\title{
Shower Counter Resolution Scaling
}

\author{
Thomas B. W. Kirk
}

\section{October 1991}

\section{DISCLAIMER}

\begin{abstract}
This report was prepared as an account of work sponsored by an agency of the United States Government. Neither the United States Government nor any agency thereof, nor any of their employees, makes any warranty, express or implied, or assumes any legal liability or responsibility for the accuracy, completeness, or usefulness of any information, apparatus, product, or process disclosed, or represents that its use would not infringe privately owned rights. Reference herein to any specific commercial product, process, or service by trade name, trademark, manufacturer, or otherwise does not necessarily constitute or imply its endorsement, recommendation, or favoring by the United States Government or any agency thereof. The views and opinions of authors expressed herein do not necessarily state or reflect those of the United States Government or any agency thereof.
\end{abstract}




\title{
Shower Counter Resolution Scaling
}

\author{
Thomas B. W. Kirk \\ Argonne National Laboratory \\ October 14, 1991
}

\begin{abstract}
The EM shower counter for the SDC detector has a resolution expression containing two stochastic terms plus a constant term. Recent measurements clarifying the sources of these terms are presented here.
\end{abstract}

\section{Introduction}

The intrinsic energy resolution of a sampling-type electromagnetic (EM) shower counter (such as the one chosen for the SDC detector) is governed by four conditions:

1) average radiation length per unit mass $\left(Z^{2} / A\right)$ of the composite materials making up the counter,

2) longitudinal granularity of sampling (i.e. the unit cell thickness in $\mathrm{X}_{0}$ );

3) mass ratios of active to passive materials in the unit cell,

4) quality control tolerances maintained over all components (dimensions, uniformity, chemistry, assembly, etc.) as used in the final device.

The first three items control the stochastic term in the resolution expression and the last item plays a major role in controlling the constant term. We do not consider the issue of calibration here but this also has a major effect on the realizable energy resolution..

In this note we assume a shower counter constructed of alternating plates of metallic $\mathrm{Pb}$ and scintillator plastic with optical fiber readout. This choice has already been made for SDC and what remains to be determined is the specific unit cell prescription.

We also carry out this discussion in terms of normally incident electrons and photons, but remind the reader that the physically relevant variable is $\mathrm{E}_{\mathrm{T}}$. The resolution in $\mathrm{E}_{\mathrm{T}}$ scales essentially with $1 / \sin \theta$ in the same manner as do the geometric properties of a shower counter with all plates oriented parallel to the beam direction (as in the SDC barrel). Thus, a shower with a given total energy resolution at $90^{\circ}$ will have the same resolution in $E_{T}$ throughout the barrel for this geometry. The endcap resolution can be chosen independently in the SDC design and is not explicitly addressed in this note.

Most of these facts are well known to SDC members and won't be further elaborated in this note. Possibly less well known is the fact that the so-called 'stochastic 
term in the resolution equation actually consists of two separately controllable component terms. These terms make statistically independent contributions to the overall resolution. The two terms derive from: intrinsic shower fluctuations which determine the fractions of energy deposited in active and passive materials; and net photoelectron yield at the photodetector. This note will concentrate on the practical implications of the two sources of stochastic resolution.

The expression for the EM shower energy resolution is given here in Eq. 1).

1)

$$
\sigma^{2}(E)=\frac{a_{s}^{2}+a_{\gamma}^{2}}{E}+b^{2} \equiv \frac{a^{2}}{E}+b^{2}
$$

where; $\quad a_{\mathrm{s}}=$ resolution constant for EM shower fluctuations in the active medium (scintillator)

$\mathrm{a}_{\gamma}=$ resolution constant for photoelectron statistics

$\mathrm{a}=$ measured effective stochastic resolution constant

$\mathrm{b}=$ constant term coefficient

From this equation, we identify the terms referred to above.

A recent beam test of the ANL/WSTC EM Test Module allows a useful separation of the shower and photoelectron source terms in the stochastic coefficient. In the beam test, electrons with energy $19 \mathrm{GeV}$ and $33 \mathrm{GeV}( \pm 1.5 \%)$ were input on the shower counter. Transverse (and longitudinal) shower containment at the center of the test module towers was excellent, so good tests of resolution were possible. These tower-centered measurements are the relevant ones for the analysis presented in this note.

Application of a $2.6 \mathrm{X}$ neutral density filter in the path of optical photons (traveling to the photocathode of the PMT viewing the shower light) allowed separation of the stochastic term due to photoelectron statistics from the intrinsic shower fluctuation term.

The measured test beam results can be used to compare the test module with energy resolution results measured for the CDF shower counter. The two stochastic terms for the CDF system have also been separately measured. All the data taken together for the test module and the CDF EM calorimeter allow good predictability for optimizing the design of the SDC EM shower counters, an exercise that must be completed in the near future.

\section{Test Module Description}

The ANL/WSTC Test Module 1 was constructed of twenty-six $5.0 \mathrm{~mm}$ thick $\mathrm{Pb}$ plates of $10 \mathrm{~cm} \mathrm{x} 10 \mathrm{~cm}$ transverse size interleaved with $2.5 \mathrm{~mm}$ scintillator plates of Bicron Type RH4 scintillator (an experimental radhard scintillator plastic with good light yield and moderate radhardness).

The scintillator light was read out by a $1.0 \mathrm{~mm}$ diameter wavelength shifter fiber of Bicron Type 91A spliced to clear $1.0 \mathrm{~mm}$ diameter Kuraray optical fiber, immediately 
outside the tile's fiber groove. Double-ended wavelength-shilır optical fibers were optically connected to the PMT from each tile using a pair of clear fibers.

We summarize the relevant mechanical parameters for the test module as:

$$
\begin{aligned}
& t_{P b}(T M)=5.0 \mathrm{~mm} \mathrm{~Pb}, \\
& t_{\text {Sin }}(T M)=2.5 \mathrm{~mm} \text { Bicron RH4 } \\
& w_{P b}(T M)=5.675 \mathrm{gm} / \mathrm{cm}^{2} \\
& w_{S c i n}(T M)=0.26 \mathrm{gm} / \mathrm{cm}^{2}
\end{aligned}
$$

Earlier bench tests using $3 \mathrm{MeV}$ electrons had shown good transverse and longitudinal uniformity for the tiles used in the test module. As a result, the constant term in the resolution expression was expected to be small. At the beam energies used, the stochastic term numerically dominates the constant term in the test module (as measured).

Bench tests on the variation of light yield with scintillator plate thickness (with constant optical shifter fiber diameter of $1 \mathrm{~mm}$ ) for minimum-ionizing particles are consistent with a parametrization given by:

$$
f=\left(t_{\text {scin }} / 2.5 \mathrm{~mm}\right)^{2 / 3}
$$

The photoelectron yield for traversal of $3 \mathrm{MeV}$ electrons from a Ru106 source with the RH4 tiles was measured to be:

$$
\mathrm{n}_{\min }=3.0 \text { photoelectrons per plate per mip }
$$

The test module was mounted in the MP charged particle beam at Fermilab so that charged particles with variable energies tagged to $1.5 \%$ and verified as electrons with a pair of beamline Cerenkov counters, could be used to measure the shower counter response $(\mathrm{E} / \mathrm{P})$. Runs at $19 \mathrm{GeV}$ and $33 \mathrm{GeV}$ were used in the present analysis.

\section{Data and Analysis}

The measured test module response to tagged incident electrons is displayed in Figure 1. The data are also reproduced here in Table I. 
Table I

Test Module Measurements in the Fermilab MP Beam

\begin{tabular}{|c|c|c|}
\hline $\begin{array}{c}E_{\text {beam }} \\
(G e V)\end{array}$ & $\sigma(\Delta E / E)$ & Comment \\
\hline 33 & 0.036 & no optical attenuation \\
\hline 19 & 0.046 & no optical attenuation \\
\hline 33 & 0.0415 & $2.6 \mathrm{X}$ optical attenuation \\
\hline 19 & 0.0525 & $2.6 \mathrm{X}$ optical attenuation \\
\hline
\end{tabular}

These measured points were fit to determine the parameters of the resolution function in Equation 1). We note that the beam tagging system has a resolution of about $1.5 \%$ which is independent of beam momentum. With the measurements made here, the contribution of the beam momentum spread cannot be separated from the intrinsic resolution term arising from manufacturing tolerances in the test module parts. From the numerical value found for $b$, it is clear that the manufacturing tolerance contribution is small compared to $1 \%$. This is consistent with the known properties of the test module as fabricated.

Eq. 1), we find:

Solving the set of simultaneous equations using the data points together with

$$
\begin{array}{ll}
a_{s}=0.167 & \text { shower statistics coefficient } \\
a_{\gamma}=0.090 & \text { photoelectron coefficient } \\
a=0.190 & \text { net stochastic term coefficient } \\
\mathrm{b}=0.0135 & \text { constant term }
\end{array}
$$

These parameters (except for $b$ ) are accurate to about $5 \%$ of their derived values.

From the fit parameters, we can make some useful observations. Since the value of ay represents the reciprocal square root of the number of photoelectrons at $1 \mathrm{GeV}$, we note for the test module:

$$
N_{\gamma}=1 / a_{\gamma}^{2}=123 \text { photoelectrons } / \mathrm{GeV}
$$

We further note that the stochastic resolution constant is dominated by shower statistics rather than by photoelectrons. The implication here is that finer longitudinal sampling could make significant improvements in the overall resolution, even with no change in the per $\mathrm{GeV}$ photoelectron yields. 
Tile/Fiber EMC Resolution

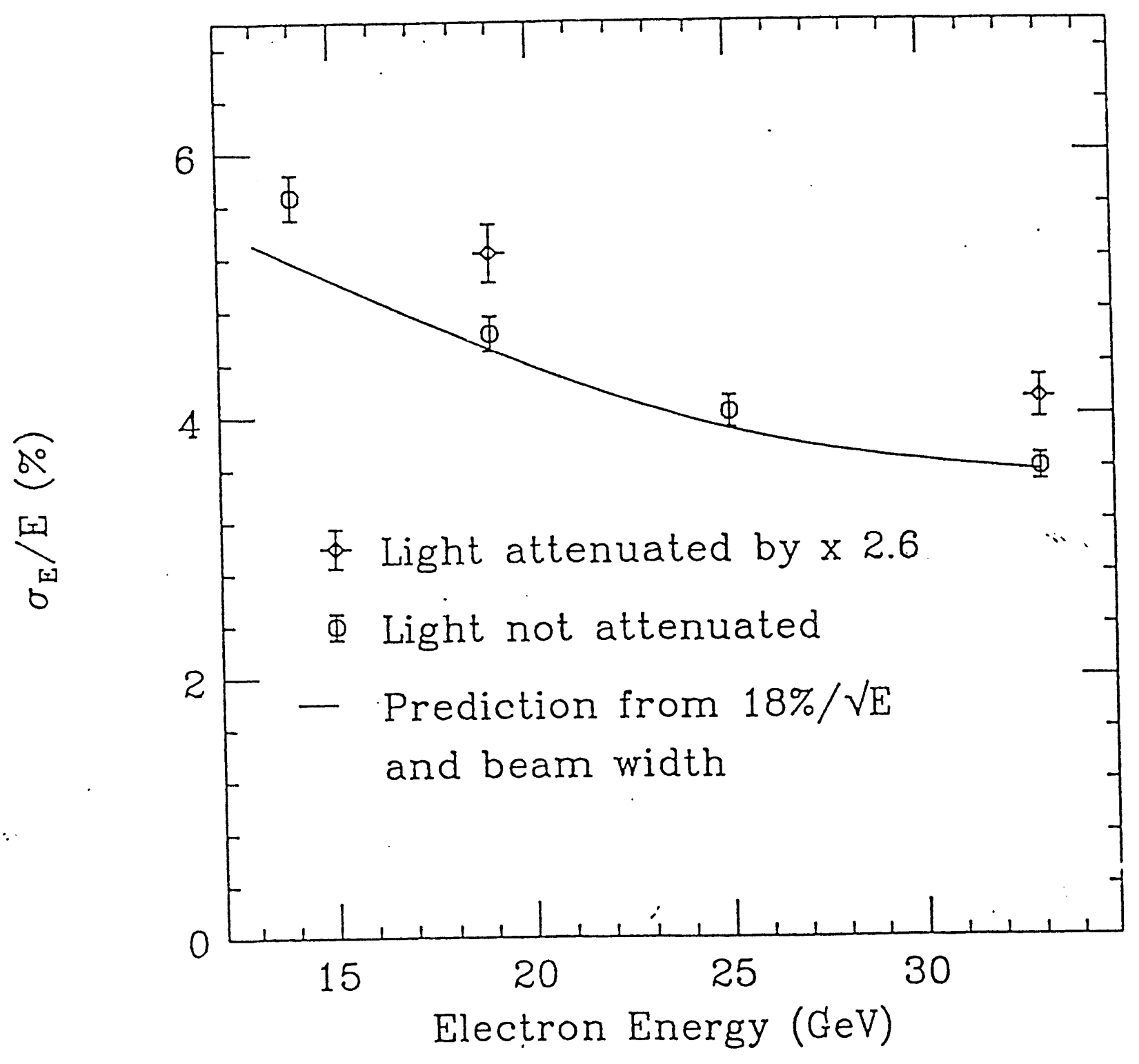

Figure 1: Measurements of resolution on the ANL/WSTC Test Module in the Fermilab MP Test Beam. 
Before applying what we have learned here to the general SDC design question, we go on to quote a similar analysis made for the CDF EM shower counter.1

$$
\sigma_{c}^{2}(e)=\frac{a_{c s}^{2}+a_{c \gamma}^{2}}{E}+b_{c}^{2} \equiv \frac{a_{c}^{2}}{E}+b_{c}^{2}
$$

where;

$$
\begin{array}{ll}
\mathrm{a}_{\mathrm{cs}}=0.116 & \text { shower statistics coefficient } \\
\mathrm{a}_{\mathrm{c} \gamma}=0.071 & \text { photoelectron statistics coefficient } \\
\mathrm{a}=0.135 & \text { net stochastic term coefficient } \\
\mathrm{b}_{\mathrm{c}} \leq 0.02 & \text { constant term }
\end{array}
$$

giving;

$$
N_{c \gamma}=1 / a_{c \gamma}^{2}=200 \text { photoelectrons } / \mathrm{GeV} \text {. }
$$

These parameters are measured for the actual CDF geometry which consists of: 2

$$
\begin{aligned}
t_{c P b}= & 3.175 \mathrm{~mm} \text { rolled } \mathrm{Pb} \text { plate } \\
& \text { (with } 0.76 \mathrm{~mm} \text { Al sheet ciadding) } \\
t_{c S c i n}= & 5.0 \mathrm{~mm} S C S N-38 \text { Plastic Scintillator } \\
w_{c P b}= & 3.60 \mathrm{gm} / \mathrm{cm}^{2} \\
w_{c S c i n}= & 0.52 \mathrm{gm} / \mathrm{cm}^{2}
\end{aligned}
$$

In addition, the per-plate photoelectron yield for minimum-ionizing tracks through the CDF shower counter has been measured to be ${ }^{1}$ :

10) $n_{c \min }=\left(\frac{300 \mathrm{MeV}}{31 \text { plates }}\right) N_{c \gamma}=1.94$ photoelectrons per plate per mip

\section{EM Shower Counter Resolution Scaling}

We can now combine the scaling rules and measurements together into a parametrization of the resolution properties to be expected of a $\mathrm{Pb} / \mathrm{S}$ cintillator $\mathrm{EM}$ shower counter with varying plate thickness. Basing the parametrization on the test model and CDF results obtained above, we derive the scaling formula for the $i^{\text {th }}$ set of parameters to be:

$$
\begin{aligned}
& \sigma_{i}^{2}(E)=\frac{a_{S i}^{2}+a_{\gamma i}^{2}}{E}+b_{i}^{2} \\
& a_{S i}^{2}=\left(\frac{t_{P b i}}{t_{P b}}\right)\left(\frac{t_{S c i n}}{t_{S c i n} i}\right)^{\alpha} a_{s}^{2}
\end{aligned}
$$




$$
\begin{aligned}
a_{\gamma}^{2} & =\left(\frac{t_{P b i}}{t_{P b}}\right)\left(\frac{t_{S c i n}}{\kappa_{i} t_{S c i n} i}\right)^{2 / 3} a_{\gamma}^{2} \\
b_{i}^{2} & \cong b^{2} \leq 0.02 \\
\kappa_{i} & =\frac{n_{\min i}}{n_{\min }}
\end{aligned}
$$

$\kappa_{i}$ is the optical efficiency, given as the ratio of photoelectrons per mip per unit thickness for the $i^{\text {th }}$ combination of scintillator and optical shifter fiber relative to the values measured for the test module tile/fiber assemblies.

There are no simple scaling rules for the constant parameter, but careful manufacture and calibration should keep this term at or below the $1 \%$ level. value of $\alpha$ :

Combining the test module and CDF data with equation 11), we can solve for the

$$
\begin{aligned}
& \alpha=\frac{\ln \left[a_{S i}^{2} t_{P b} / a_{s}^{2} t_{P b i}\right]}{\ln \left[t_{S c i n} / t_{S c i n i}\right]} \\
& \alpha=\frac{\ln \left[(.116)^{2}(5.0) /(.167)^{2}(3.175)\right]}{\ln [2.5 / 5.0]}=0.40
\end{aligned}
$$

Figure 2 shows the result of applying these scaling rules to the $\mathrm{Pb} /$ scintillator geometries of interest. The $\mathrm{Pb}$ plate thickness is the abscissa and the scintillator plate thickness is given parametrically. On the same graph are shown the measured points for the Test Module and the CDF shower counter. In Figures 3 and 4, the separate contributions of shower statistics and photoelectrons are plotted for 2.5 and $4.0 \mathrm{~mm}$ scintillator, respectively.

A principal concern of the SDC is the effect of radiation damage on the resolution of the shower counter. It has been hypothesized that we should plan for radiation damage causing loss of light of up to a factor of two while retaining the desired energy resolution. For this reason, the effect of losing a factor of two in the detected light at the PMT has been plotted along with the undamaged detector parameters.

The results look very promising. In general, the resolution deteriorates by only about $10 \%$ after losing a factor of two in light. This derives, of course, from the dominance of the energy fluctuation contribution over the photostatistics in the overall resolution.

It appears that a stochastic term level of $15 \%$ can achieved for a practical design by using $4.0 \mathrm{~mm} \mathrm{~Pb}$ plates and $4.0 \mathrm{~mm}$ scintillator. Such a design could stand a factor of two light loss while retaining $16.7 \%$ net resolution. This is well within the calorimeter specifications for SDC. 


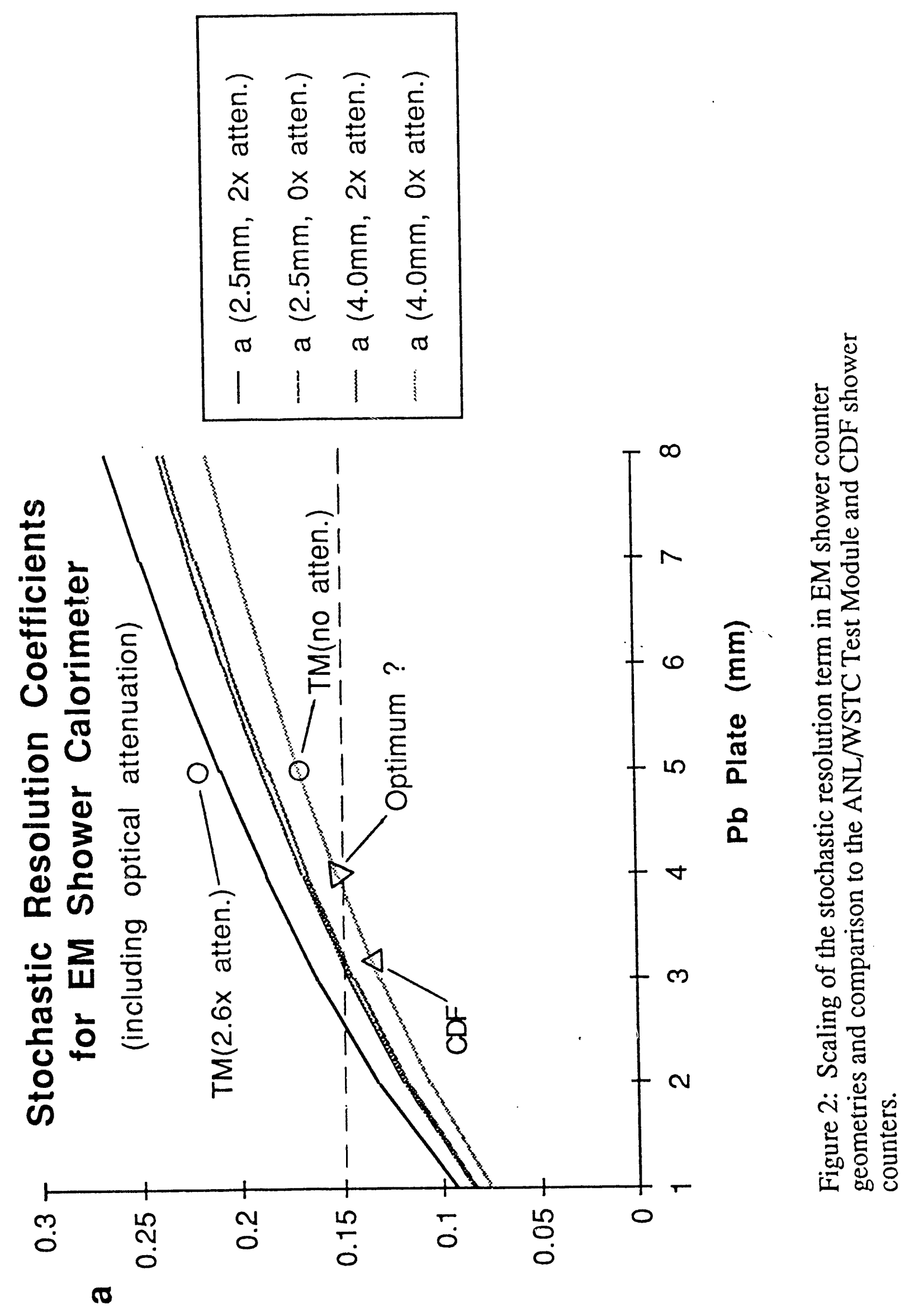




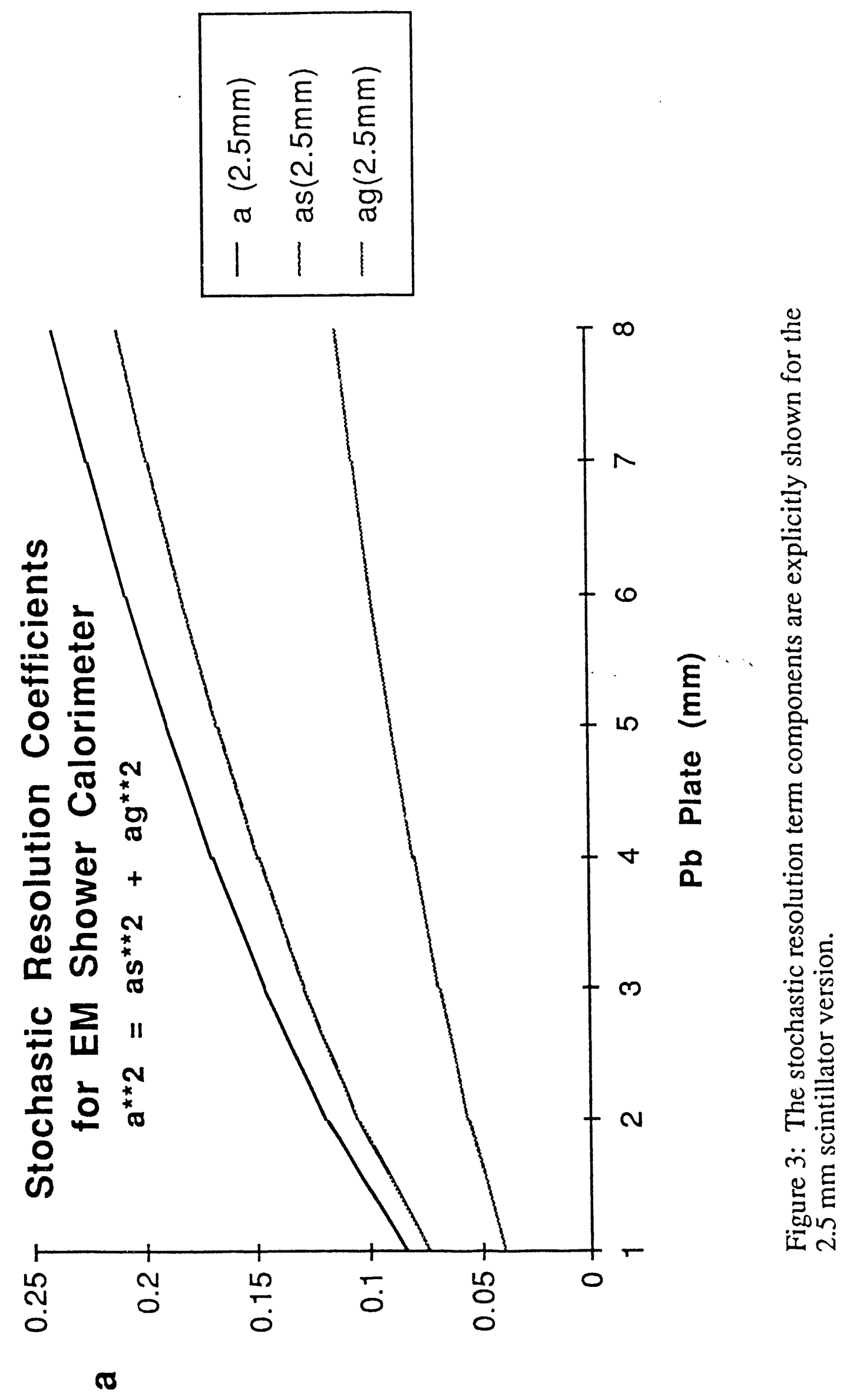




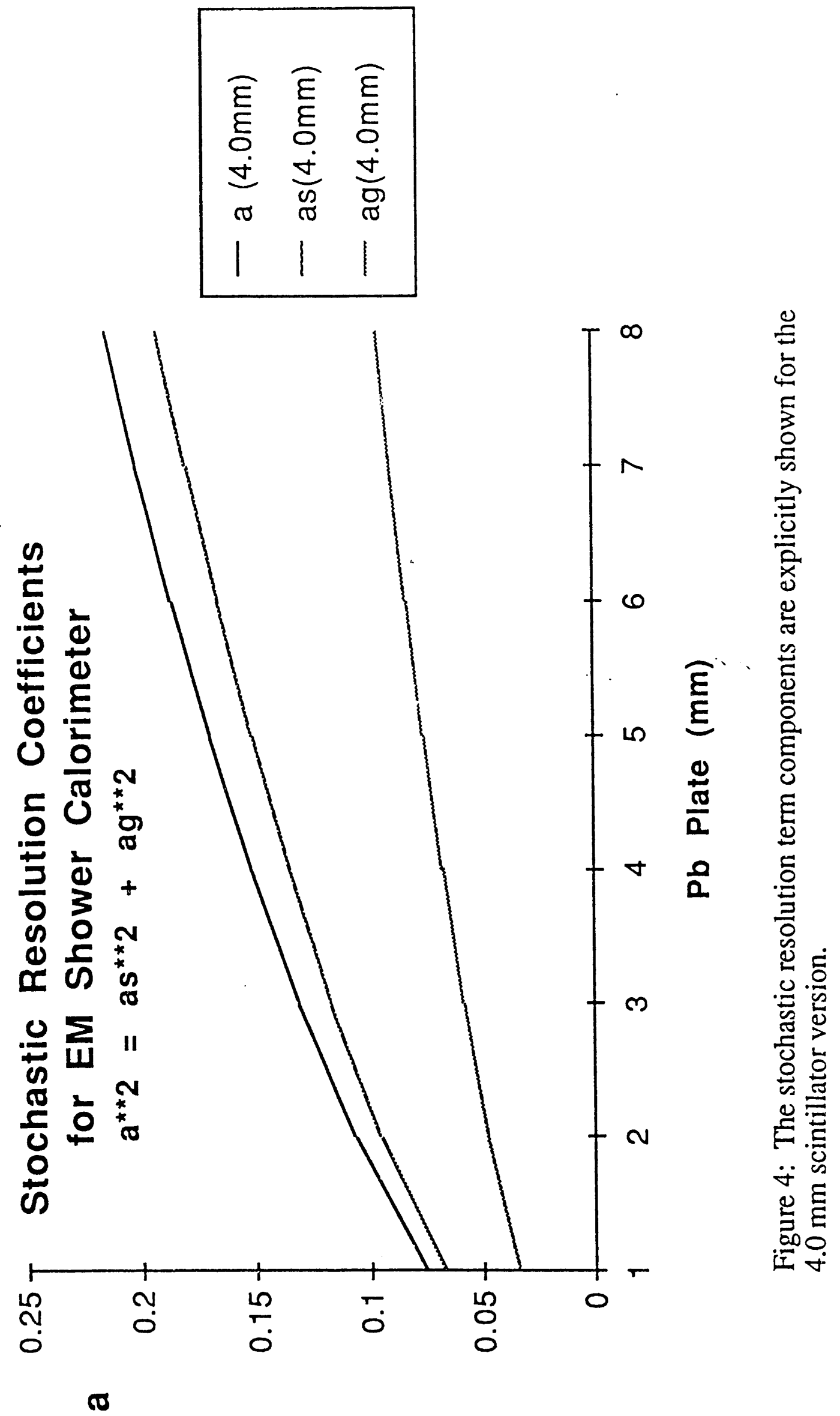




\section{Cost Considerations}

All variations of the design parameters will have cost implications. Some parameters are more benign than others. We already know that piece counts are stronger cost drivers than material weights. Also, the scintillator, and the mechanical structures account for comparable fractions of the cost. The radius of the barrel is also a sensitive cost driver of the whole detector (principally, via the muon system costs).

The increase in tile thickness from $2.5 \mathrm{~mm}$ to $4.0 \mathrm{~mm}$ will add a total of $4.0 \mathrm{~cm}$ to the radius of the Model B EM (with its $4.0 \mathrm{~mm} \mathrm{~Pb}$ plates); it will add $5.1 \mathrm{~cm}$ in Model A (with its 3.175 run $\mathrm{Pb}$ plates). ${ }^{3}$ If $4.0 \mathrm{~mm}$ tiles are used in all layers of the Model B calorimeter (probably a good idea in general), the barrel radius will be increased by $9.7 \mathrm{~cm}$.

The August 1991 cost estimate for the calorimeter used $4.0 \mathrm{~mm} \mathrm{~Pb}$ plates and 2.5 $\mathrm{mm}$ scintillator tiles. Increasing the tile thickness to $4.0 \mathrm{~mm}$ will have very small cost implications (unchanged piece -sunt, small materials cost increment, no management cost change) for the calorimeter scintillators. It will have a moderate volume cost impact by increasing the radius of the calorimeter, hence the absorber materials and machining costs. The fractional radius change (for all tiles changed to $4.0 \mathrm{~mm}$ ) is $9.7 / 450=2.2 \%$. The volume change would be $4.6 \%$. The cost change for the absorber should be less than $\$ 2.0 \mathrm{M}$.

The cost change induced by varying the Pb plate thickness is steeper due to its direct ratio relative to the scintillator piece count. For constant total EM counter thickness, the piece count goes up by the inverse ratio of $\mathrm{Pb}$ plate thicknesses. If $3.0 \mathrm{~mm} \mathrm{~Pb}$ plates are chosen, the EM tile count factor goes up by 4.0/3.0 =1.33. The cost increase will be about $\$ 2.0 \mathrm{M}$ in incremental tile costs and $\$ 1.0 \mathrm{M}$ in radial size increase (lower average density).

A serious qualitative concern with $\mathrm{Pb}$ plate thinner than $4.0 \mathrm{~mm}$ has to do with creep in the cast $\mathrm{Pb}$ design (the basis of all cost estimates so far produced). The maximum stresses in the $\mathrm{Pb}$ plates go linearly with plate thickness. For $4.0 \mathrm{~mm}$ pure $\mathrm{Pb}$ plates, the maximum stress is 62 psi, about the maximum practical value for a 20 -year detector lifetime. If thinner plates are chosen, strengthening alloy would have to be used in place of pure $\mathrm{Pb}$ to set a higher creep stress limit. In this case, costs would again rise and we would need additional $\mathrm{R} \& \mathrm{D}$ to establish the technical feasibility of casting $\mathrm{Pb}$ alloy successfully. All this can be done, but it will take time.

Pulling all the factors together, it would seem that an optimum cost/effectiveness design for the barrel EM calorimeter would one be based on $4.0 \mathrm{~mm} \mathrm{~Pb}$ plates with $4.0 \mathrm{~mm}$ scintillator tiles.

Work supported by the U.S.Department of Energy, Division of High Energy Physics, under contract W-31-109-ENG-38.

\section{REFERENCES}

1 CDF shower counter parameters were quoted by A. B. Wicklund based on current analysis of data.

2 N. Hill, ANL design engineer for CDF EM calorimeter.

3 Calorimeter Conceptual Design, Tile/ - - iber Scintillator Option, Volume 1, SDC Tile/Fiber Calorimeter Group, September 3, 1991. 

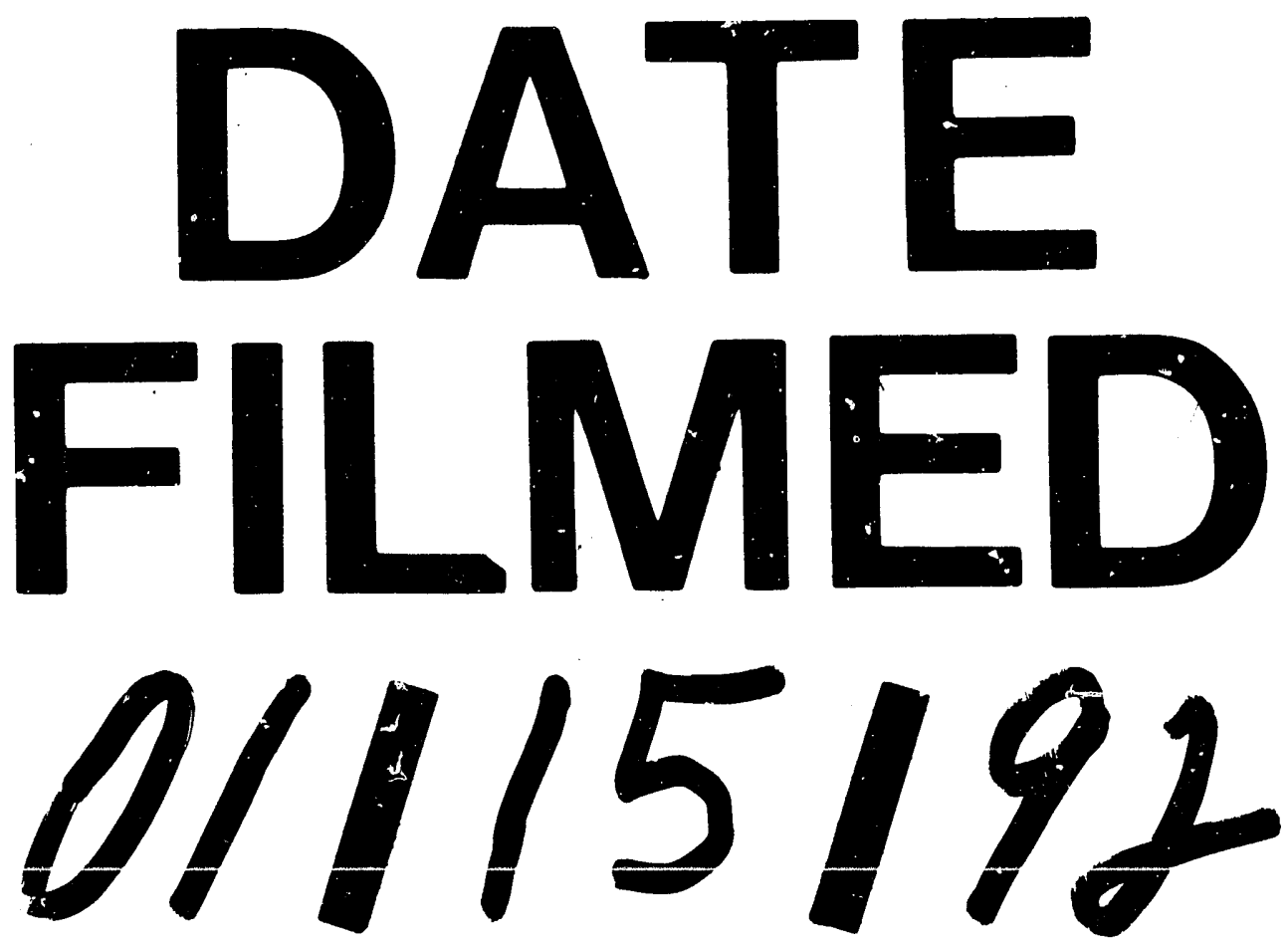
